\title{
Synthesis of BODIPY-labeled sphingosine derivatives by cross-metathesis reaction
}

Carsten Peters, Andreas Billich, Michael Ghobrial, Klemens Högenauer, Thomas Ullrich and Peter Nussbaumer ${ }^{*}$

\section{Supporting Information:}

(S)-4-[(E)-(R)-1-Hydroxy-12-(7-nitro-benzo[1,2,5]oxadiazol-4-ylamino)-dodec-2-enyl]-2,2-

(S)-4-[(E)-(R)-1-Hydroxy-7-(4,4-difluoro-5,7-dimethyl-4-bora-3a,4a-diaza- $s$-indacen-3)-hept-

(E)-(2S,3R)-2-Amino-14-(7-nitro-benzo[1,2,5] oxadiazol-4-ylamino)-tetradec-4-ene-1,3-diol

(E)-(2S,3R)-2-Amino-9-(4,4-Difluoro-5,7-dimethyl-4-bora-3a,4a-diaza-s-indacen-3)-non-4- 
General. Solvents used were dried over molecular sieves or bought as absolute solvents. Flash-chromatography was performed using Biotage SP4 with cyclohexane/ ethyl acetate in various proportions as eluent. In NMR spectra, multiplicities are given as singlet (s), doublet (d), triplet (t), quadruplet (q), quintet (quin). Coupling constants are given in Hz. All commercially available compounds were used as received.

General procedure cross-metathesis. For cross-metathesis reaction Garner-allylic alcohol $(\text { anti-9) })^{*}$ and alkenylated label at a stoichiometric ratio of 1:1 to $1: 3$ were dissolved in dry $\mathrm{CH}_{2} \mathrm{Cl}_{2}$ and the resulting solution was degassed carefully. Then, under argon-atmosphere, a catalytic amount of $2^{\text {nd }}$ generation Grubbs' catalyst was added and the reaction mixture was allowed to reflux for 2-5 $\mathrm{h}$ until TLC indicated no further change in composition of the reaction mixture. Afterwards solvent was removed in vacuo and the crude material was purified by flash column chromatography.

General procedure deprotection. For removal of Boc-/acetal protection the product of the cross-metathesis reaction was dissolved at room temperature in an appropriate amount of $4 \mathrm{M}$ $\mathrm{HCl} /$ dioxane and stirred for approx. 20 min (TLC monitoring). After removal of the solvent the crude material was purified by preparative RP-HPLC and the product containing fractions were pooled and lyophilized.

*this compound was prepared according to reference 11 and separation of diastereomers was performed on normal phase HPLC using a Nucleosil100 Si $5 \mu \mathrm{m}$ column $(250 * 40 \mathrm{~mm})$ with heptane:ethylacetate 7:3 (v/v) (isocratic) as eluent at a flow rate of $30 \mathrm{ml} / \mathrm{min}$. 


\section{4,4-Difluoro-5,7-dimethyl-4-bora-3a,4a-diaza-s-indacen-3-undec-10-ene $6 \mathrm{~b}$.}

By pursuing the same strategy as described for compound $\mathbf{6 a}$, compound $\mathbf{6 b}$ was obtained as a dark red oil starting from dipyrromethene $\mathbf{5 b}$ (340 mg, $1.05 \mathrm{mmol})$; yield: $350 \mathrm{mg}$ (0.94 mmol, $89 \%) ;{ }^{1} \mathrm{H}-\mathrm{NMR}\left(500 \mathrm{MHz}, \mathrm{CDCl}_{3}\right) \delta 1.24-1.46(\mathrm{~m}, 12 \mathrm{H}), 1.72$ (quin, $J=7.6 \mathrm{~Hz}, 2 \mathrm{H}$ ), $2.03(\mathrm{q}, J=7.4 \mathrm{~Hz}, 2 \mathrm{H}), 2.24(\mathrm{~s}, 3 \mathrm{H}), 2.55(\mathrm{~s}, 3 \mathrm{H}), 2.96(\mathrm{t}, J=7.9 \mathrm{~Hz}, 2 \mathrm{H}), 4.92(\mathrm{dd}, J=10.2$ Hz, 1.1 Hz, 1H), 4.99 (dd, J=17.2 Hz, 1.9 Hz, 1H), 5.81 (ddt, J=16.6 Hz, $6.8 \mathrm{~Hz}, 6.6 \mathrm{~Hz} 1 \mathrm{H})$, $6.08(\mathrm{~s}, 1 \mathrm{H}), 6.28$ (d, J=4.0 Hz, 1H), 6.90 (d, J=4.0 Hz, 1H), 7.05 (s,1H); ESI-MS ${ }^{+}: 373.2$ [M+Na]; HRMS: M calcd for $\mathrm{C}_{22} \mathrm{H}_{32} \mathrm{BF}_{2} \mathrm{~N}_{2}$ 372.2657, found 372.2658.

(S)-4-[(E)-(R)-1-Hydroxy-12-(7-nitro-benzo[1,2,5]oxadiazol-4-ylamino)-dodec-2-enyl]2,2-dimethyl-oxazolidine-3-carboxylic acid tert-butyl ester 10a.

According to the general procedure, Garner allylic alcohol (anti-9) $(65 \mathrm{mg}, 0.25 \mathrm{mmol})$ were reacted with alkenylated-NBD dye $2(100 \mathrm{mg}, 0.30 \mathrm{mmol})$ and $2^{\text {nd }}$ gen. Grubbs' catalyst (21 mg, $0.025 \mathrm{mmol})$ : yield $95 \mathrm{mg}(0.17 \mathrm{mmol}, 66 \%)$; orange oil; ${ }^{1} \mathrm{H}-\mathrm{NMR}$ (400MHz, $\left.\mathrm{CDCl}_{3}\right) \delta 1.31(\mathrm{~s}, 6 \mathrm{H}), 1.32-1.68(\mathrm{~m}, 12 \mathrm{H}), 1.49(\mathrm{~s}, 9 \mathrm{H}), 1.81$ (quin. J= 7.8 Hz, 2H), $2.04(\mathrm{q}$, $J=7.0 \mathrm{~Hz}, 2 \mathrm{H}), 3.48(\mathrm{q}, J=7.0 \mathrm{~Hz}, 2 \mathrm{H}), 3.76-4.27(\mathrm{~m}, 4 \mathrm{H}), 5.45(\mathrm{dd}, J=15.4 \mathrm{~Hz}, 6.1 \mathrm{~Hz}$, 1H), $5.45(\mathrm{td}, J=14.1 \mathrm{~Hz}, 6.4 \mathrm{~Hz}, 1 \mathrm{H}), 6.18(\mathrm{~d}, J=8.6 \mathrm{~Hz}, 1 \mathrm{H}), 6.29(\mathrm{~s}, 1 \mathrm{H}), 8.50$ (d, J= 8.6 $\mathrm{Hz}, 1 \mathrm{H}) ;{ }^{13} \mathrm{C}-\mathrm{NMR}(125.7 \mathrm{MHz}) \delta 26.3,26.9,28.4,28.5,28.6,29.0,29.1,29.2,29.3,29.4$, 29.5, 32.3, 44.1, 94.4, 98.5, 123.6, 128.2, 129.0, 133.2, 133.8, 136.5, 143.9, 144.1, 144.3; ESI-MS ${ }^{+}: 584.4$ [M+Na]; HRMS: M calcd for $\mathrm{C}_{28} \mathrm{H}_{43} \mathrm{~N}_{5} \mathrm{O}_{7} \mathrm{Na}$ 584.3061, found 584.3054. 


\section{(S)-4-[(E)-(R)-1-Hydroxy-7-(4,4-difluoro-5,7-dimethyl-4-bora-3a,4a-diaza-s-indacen-}

\section{3)-hept-2-enyl]-2,2-dimethyl-oxazolidine-3-carboxylic acid tert-butyl ester $10 \mathrm{~b}$.}

According to the general procedure, Garner allylic alcohol (anti-9) $(111 \mathrm{mg}, 0.43 \mathrm{mmol})$ were reacted with C6-alkenyl-BDP $7(65 \mathrm{mg}, 0.22 \mathrm{mmol})$ and $2^{\text {nd }}$ gen. Grubbs' catalyst (18 $\mathrm{mg}, 0.022 \mathrm{mmol})$ : yield $77 \mathrm{mg}(0.14 \mathrm{mmol}, 79 \%)$; orange oil; ${ }^{1} \mathrm{H}-\mathrm{NMR}\left(400 \mathrm{MHz}, \mathrm{CDCl}_{3}\right) \delta$ $1.46-1.50(\mathrm{~m}, 15 \mathrm{H}), 1.53$ (quin. $J=7.7 \mathrm{~Hz}, 2 \mathrm{H}$ ), 1.75 (quin. $J=7.7 \mathrm{~Hz}, 2 \mathrm{H}), 2.12$ (q, $J=7.4$ $\mathrm{Hz}, 2 \mathrm{H}), 2.24(\mathrm{~s}, 3 \mathrm{H}), 2.55(\mathrm{~s}, 3 \mathrm{H}), 2.97(\mathrm{t}, J=7.8 \mathrm{~Hz}, 2 \mathrm{H}), 3.78-4.25(\mathrm{~m}, 4 \mathrm{H}), 5.47(\mathrm{dd}, J=$ $15.3 \mathrm{~Hz}, 6.1 \mathrm{~Hz}, 1 \mathrm{H}), 5.74(\mathrm{td}, J=15.3 \mathrm{~Hz}, 6.1 \mathrm{~Hz}, 1 \mathrm{H}), 6.08(\mathrm{~s}, 1 \mathrm{H}), 6.28(\mathrm{~d}, J=4.0 \mathrm{~Hz}, 1 \mathrm{H})$, $6.90(\mathrm{~d}, J=4.0 \mathrm{~Hz}, 1 \mathrm{H}), 7.06(\mathrm{~s}, 1 \mathrm{H}) ; \mathrm{ESI}^{-M^{+}}{ }^{+}: 554.3[\mathrm{M}+\mathrm{Na}]$.

(E)-(2S,3R)-2-Amino-14-(7-nitro-benzo[1,2,5]oxadiazol-4-ylamino)-tetradec-4-ene-1,3diol 11a.

According to the general procedure fully protected NBD-labeled sphingosine 10a $(50 \mathrm{mg}$, $0.09 \mathrm{mmol}$ ) were treated with $4 \mathrm{ml}$ of $4 \mathrm{M} \mathrm{HCl} /$ dioxane and afterwards purified; yield $26 \mathrm{mg}$ (0.06 mmol, 70\%); orange lyophilisate; ${ }^{1} \mathrm{H}-\mathrm{NMR}\left(400 \mathrm{MHz}, \mathrm{d}_{4}-\mathrm{MeOD}\right) \delta 1.28-1.33(\mathrm{~m}, 16 \mathrm{H})$, 1.75-1.83 (m, 2H), 2.10 (q $J=7.4 \mathrm{~Hz}, 2 \mathrm{H}), 3.20-3.23(\mathrm{~m}, 1 \mathrm{H}), 3.48-3.53$ (m, 1H), 3.45 (dd, $J=11.6 \mathrm{~Hz}, 4.7 \mathrm{~Hz}, 1 \mathrm{H}), 3.81(\mathrm{dd}, J=11.6 \mathrm{~Hz}, 4.0 \mathrm{~Hz}, 1 \mathrm{H}), 4.29$ (t, $J=5.4 \mathrm{~Hz}, 1 \mathrm{H}), 5.48$ (dd, $J=15.2 \mathrm{~Hz}, 6.0 \mathrm{~Hz}, 1 \mathrm{H}), 5.84(\mathrm{td}, J=14.3 \mathrm{~Hz}, 6.3 \mathrm{~Hz}, 1 \mathrm{H}), 6.35(\mathrm{~d}, J=8.9 \mathrm{~Hz}, 1 \mathrm{H}), 8.55(\mathrm{~d}$, $J=8.9 \mathrm{~Hz}, 1 \mathrm{H}) ;{ }^{13} \mathrm{C}-\mathrm{NMR}\left(125 \mathrm{MHz}, \mathrm{CDCl}_{3}\right) \delta 26.6,28.7,28.9,29.0,29.1,29.2,31.9,57.2$, 58.0, 69.6, 97.9, 127.0, 135.1, 137.0; ESI-MS ${ }^{+}: 445.1[\mathrm{M}+\mathrm{Na}]$; HRMS: M calcd for $\mathrm{C}_{20} \mathrm{H}_{31} \mathrm{~N}_{5} \mathrm{O}_{5} \mathrm{Na}$ 444.2223, found 444.2216. 


\section{(E)-(2S,3R)-2-Amino-9-(4,4-Difluoro-5,7-dimethyl-4-bora-3a,4a-diaza-s-indacen-3)-}

\section{non-4-ene-1,3-diol $11 b$.}

According to the general procedure, fully protected BDP-labeled sphingosine $\mathbf{1 0 b}(77 \mathrm{mg}$, $0.14 \mathrm{mmol}$ ) was treated with $4 \mathrm{ml}$ of $4 \mathrm{M} \mathrm{HCl} /$ dioxane and afterwards purified; yield $35 \mathrm{mg}$ (0.07 mmol, 48\%); orange lyophilisate; ${ }^{1} \mathrm{H}-\mathrm{NMR}\left(400 \mathrm{MHz}, \mathrm{DMSO}-\mathrm{d}_{6}\right) \delta 1.53$ (quin. $J=7.7$ Hz, 2H), 1.68 (quin. $J=7.7 \mathrm{~Hz}, 2 \mathrm{H}) 2.06(\mathrm{q} J=7.3 \mathrm{~Hz}, 2 \mathrm{H}), 2.25(\mathrm{~s}, 3 \mathrm{H}), 2.43(\mathrm{~s}, 3 \mathrm{H}), 2.83(\mathrm{t}$, $J=7.7 \mathrm{~Hz}, 2 \mathrm{H}), 2.97-3.06(\mathrm{~m}, 1 \mathrm{H}), 3.45(\mathrm{dd}, J=11.3 \mathrm{~Hz}, 2.9 \mathrm{~Hz}, 1 \mathrm{H}), 3.59(\mathrm{dd}, J=11.3 \mathrm{~Hz}$, $4.1 \mathrm{~Hz}, 1 \mathrm{H}), 4.17$ (dd, $J=9.2 \mathrm{~Hz}, 4.5 \mathrm{~Hz}, 1 \mathrm{H}), 5.03-5.19$ (bs, 2H), 5.45 (dd, J= 15.3 Hz, 6.6 $\mathrm{Hz}, 1 \mathrm{H}), 5.74(\mathrm{td}, J=15.3 \mathrm{~Hz}, 6.9 \mathrm{~Hz}, 1 \mathrm{H}), 6.27(\mathrm{~s}, 1 \mathrm{H}), 6.40(\mathrm{~d}, J=3.9 \mathrm{~Hz}, 1 \mathrm{H}), 7.10$ (d, J=

$3.9 \mathrm{~Hz}, 1 \mathrm{H}), 7.66$ (s, 1H), 7.74 (bs, 3H); ${ }^{13} \mathrm{C}-\mathrm{NMR}(100 \mathrm{MHz}) \delta 11.2,14.6,27.8,27.9,28.5$, 31.4, 57.0, 58.1, 69.0, 117.0, 120.2, 125.3, 128.7, 129.3, 132.9, 133.0, 134.3, 143.8, 158.6, 159.3; ESI-MS ${ }^{+}$: 392.2 [M+H]; HRMS: $\mathrm{M}$ calcd for $\mathrm{C}_{20} \mathrm{H}_{28} \mathrm{BF}_{2} \mathrm{~N}_{3} \mathrm{O}_{2} \mathrm{Na}$ 413.2171, found 413.2171. 
4,4-Difluoro-5,7-dimethyl-4-bora-3a,4a-diaza-s-indacen-3-hex-5-ene $\quad \mathbf{6 a}$

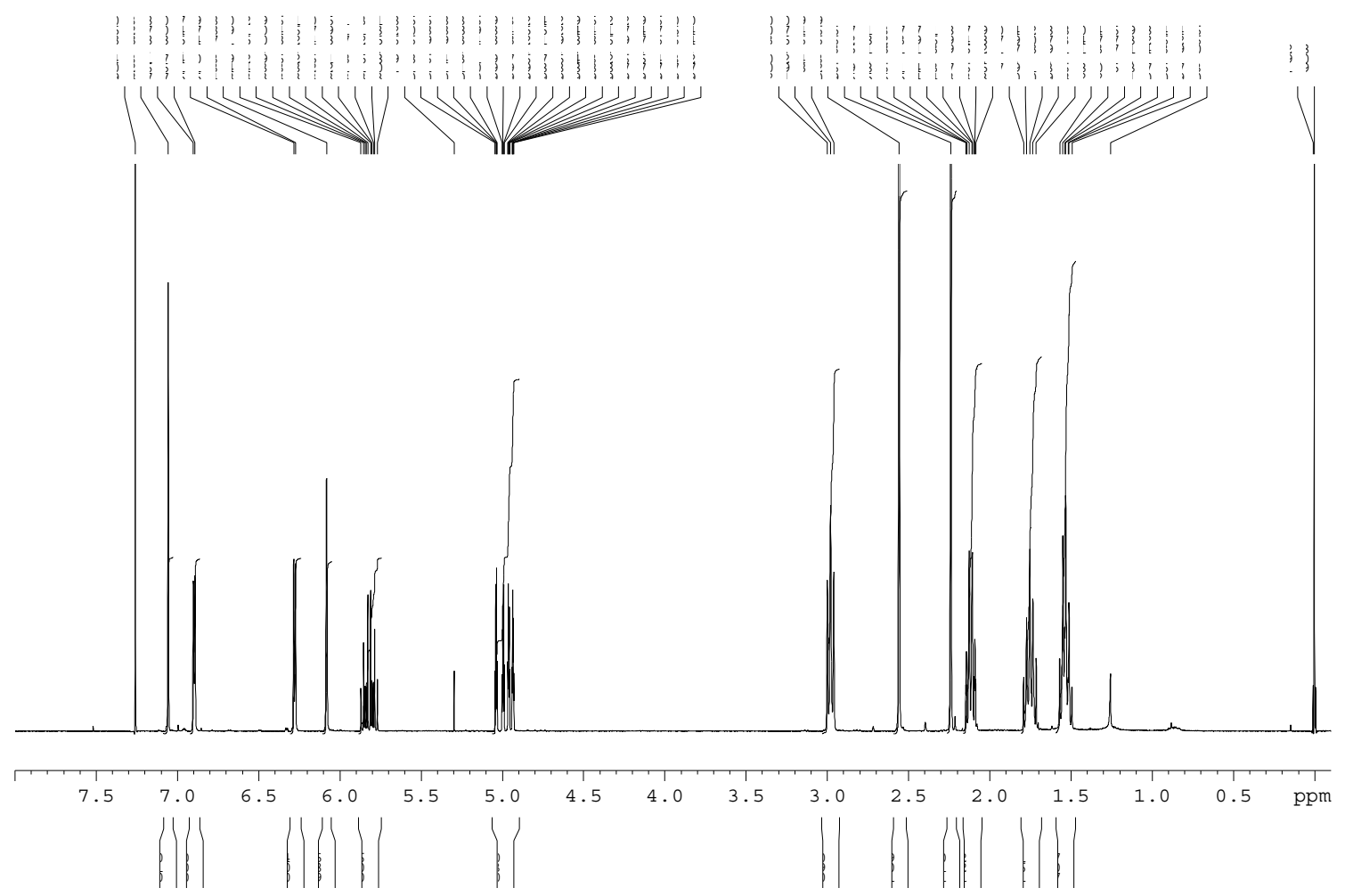

4,4-Difluoro-5,7-dimethyl-4-bora-3a,4a-diaza-s-indacen-3-undec-10-ene $\mathbf{6 b}$ 


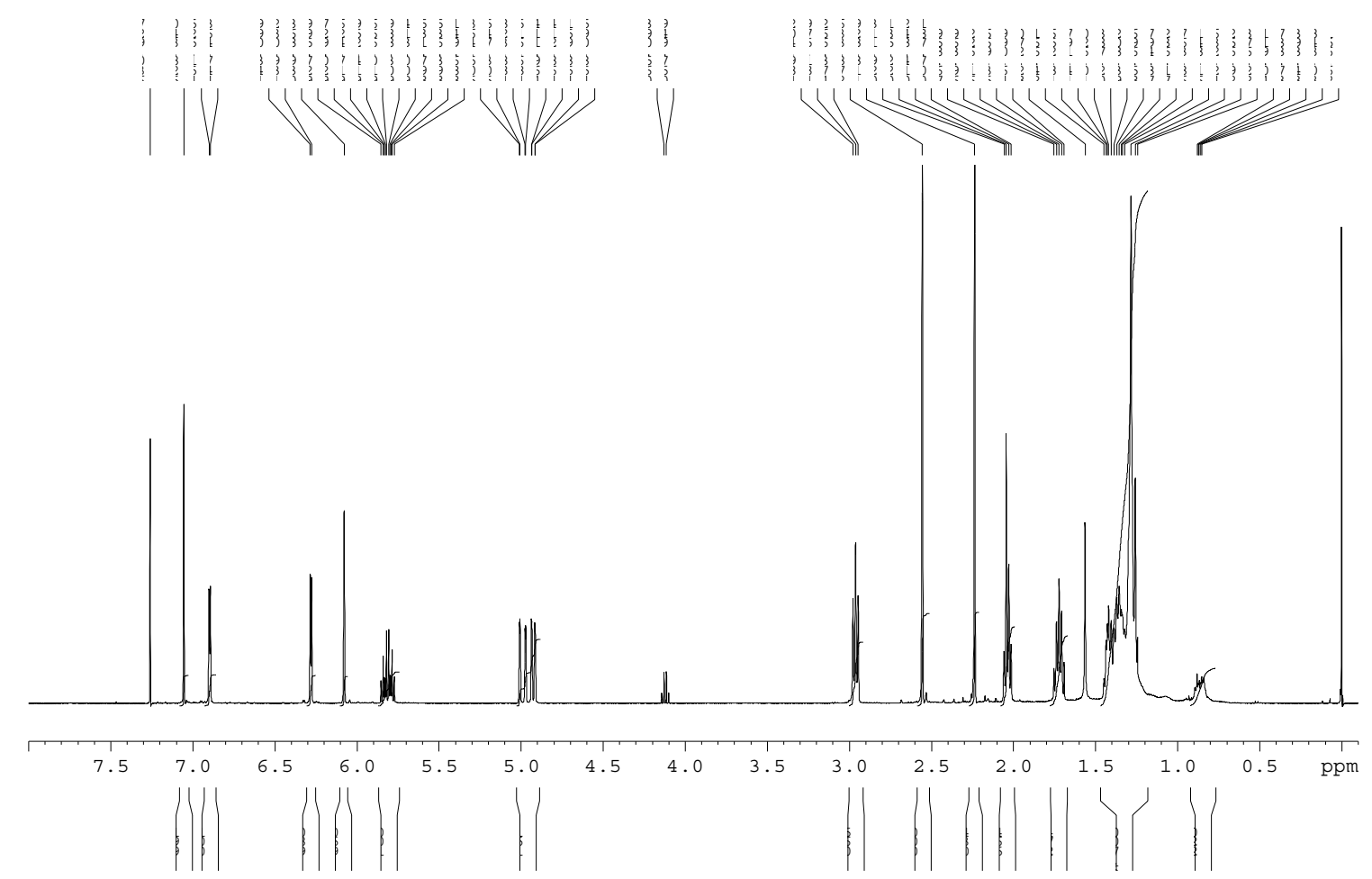

\section{4,4-Difluoro-1,3,5,7-tetramethyl-8-(dec-9-en)-4-bora-3a,4a-diaza-s-indacene 7}

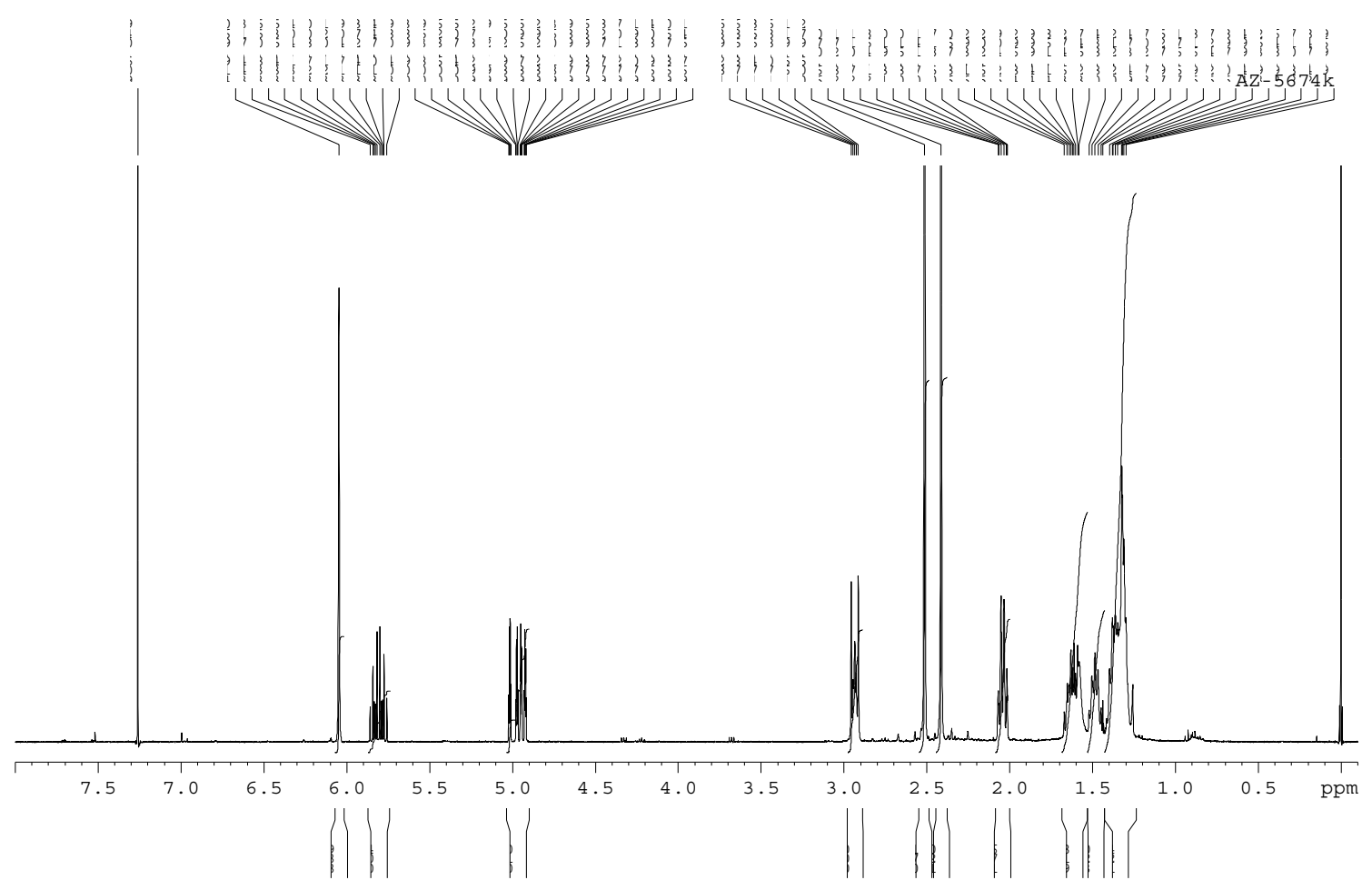

(S)-4-[(E)-(R)-1-Hydroxy-12-(7-nitro-benzo[1,2,5]oxadiazol-4-ylamino)-dodec-2-enyl]-

2,2-dimethyl-oxazolidine-3-carboxylic acid tert-butyl ester 10a. 


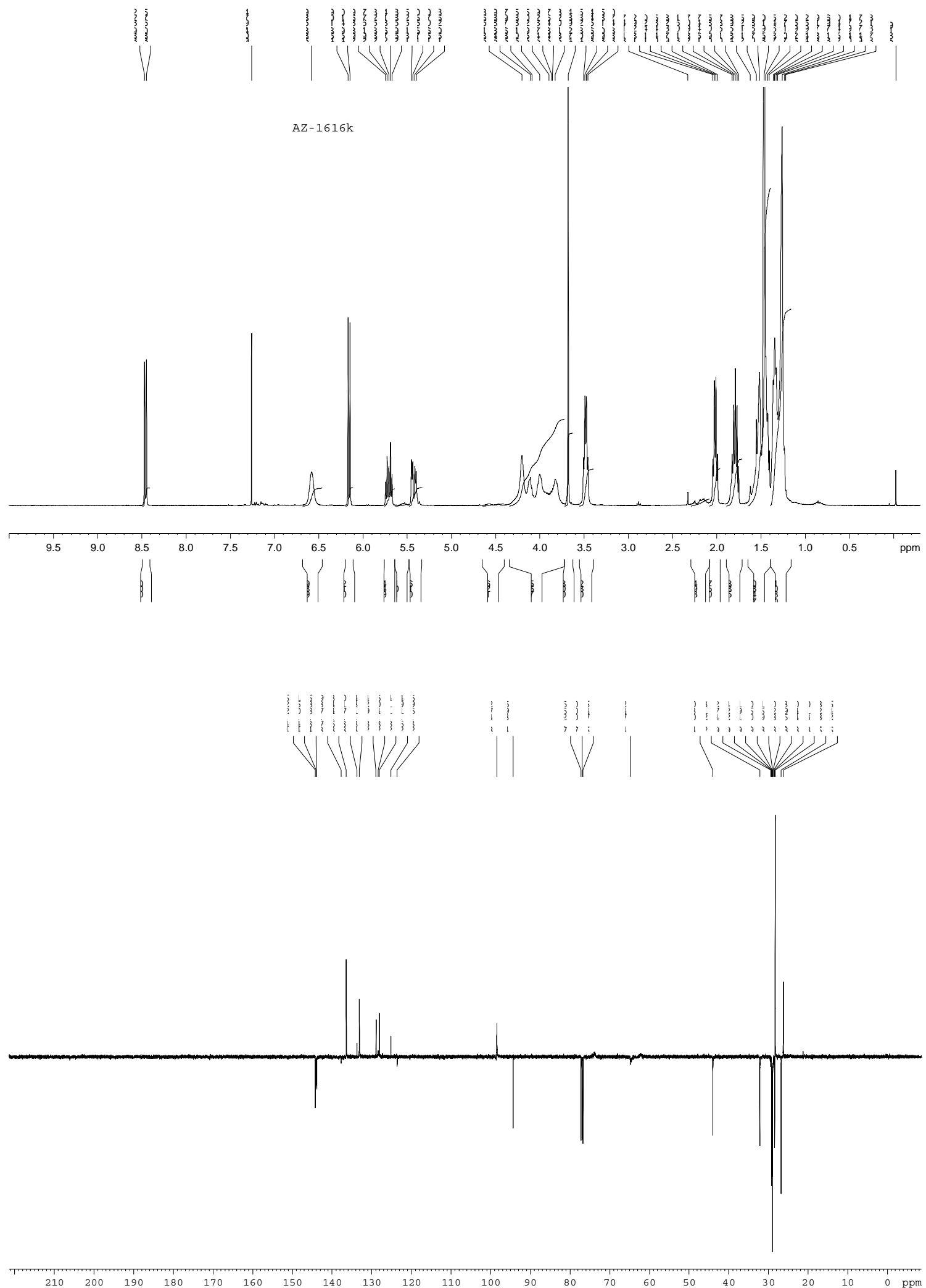

(S)-4-[(E)-(R)-1-Hydroxy-7-(4,4-difluoro-5,7-dimethyl-4-bora-3a,4a-diaza-s-indacen-3)-

hept-2-enyl]-2,2-dimethyl-oxazolidine-3-carboxylic acid tert-butyl ester $\quad \mathbf{1 0 b}$ 


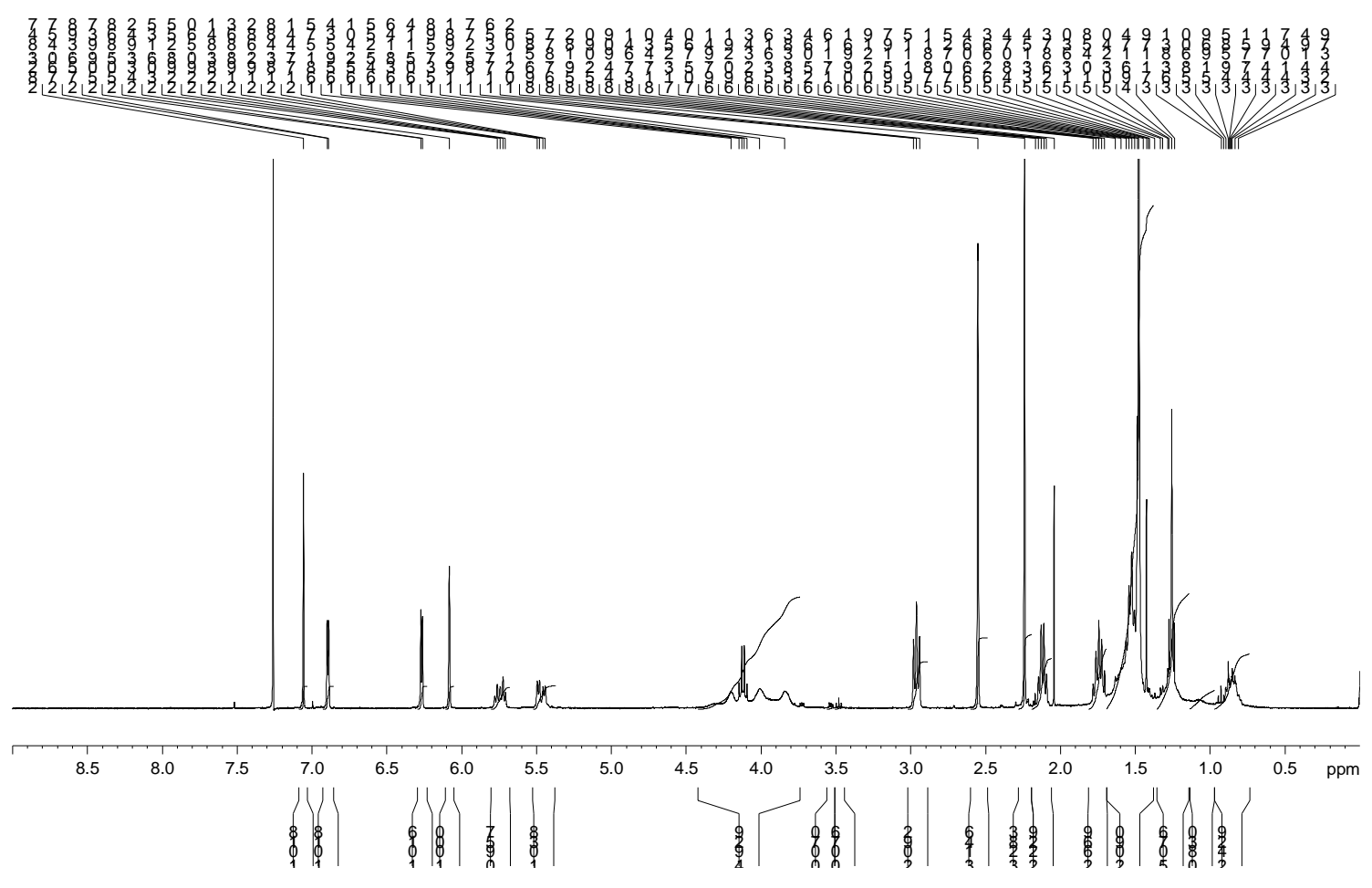

(S)-4-[(E)-(R)-1-Hydroxy-12-(4,4-difluoro-5,7-dimethyl-4-bora-3a,4a-diaza-s-indacen-3)dodec-2-enyl]-2,2-dimethyl-oxazolidine-3-carboxylic acid tert-butyl ester 10c

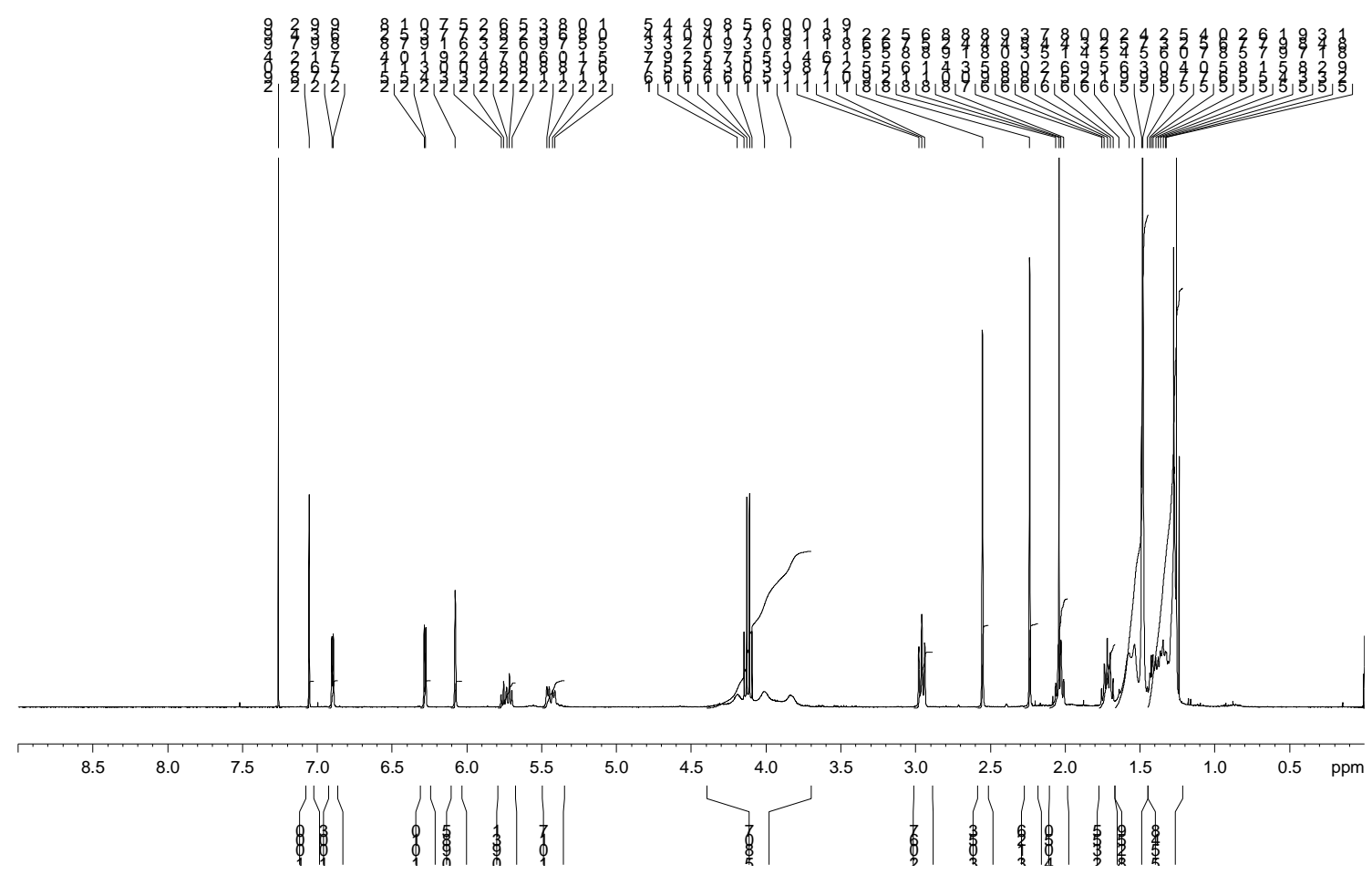


(S)-4-[(E)-(R)-1-Hydroxy-11-(4,4-difluoro-1,3,5,7-tetramethyl-4-bora-3a,4a-diaza-sindacene-8)-undec-2-enyl]-2,2-dimethyl-oxazolidine-3-carboxylic acid tert-butylester 10d

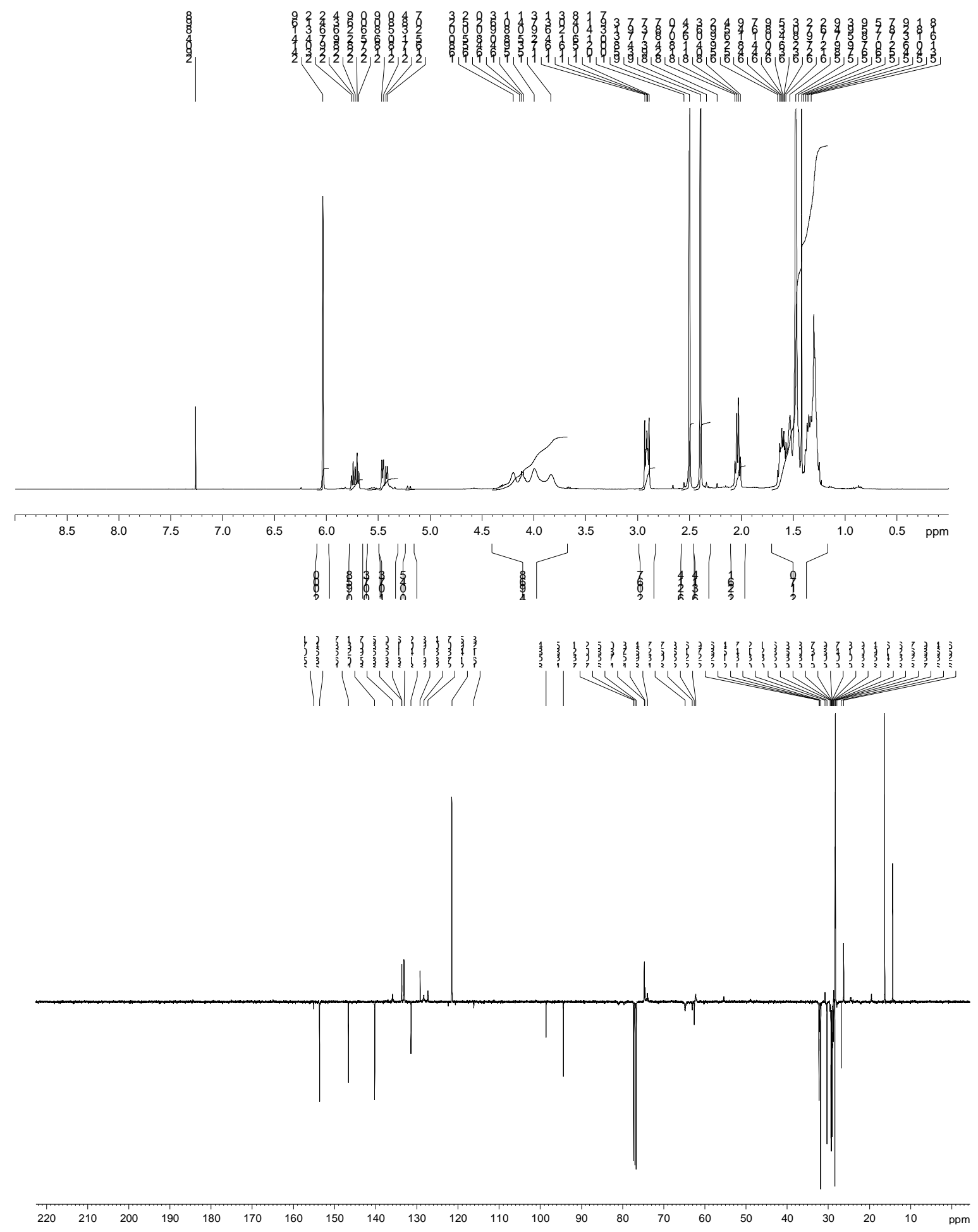


(E)-(2S,3R)-2-Amino-14-(7-nitro-benzo[1,2,5] oxadiazol-4-ylamino)-tetradec-4-ene-1,3-diol

\section{1a}
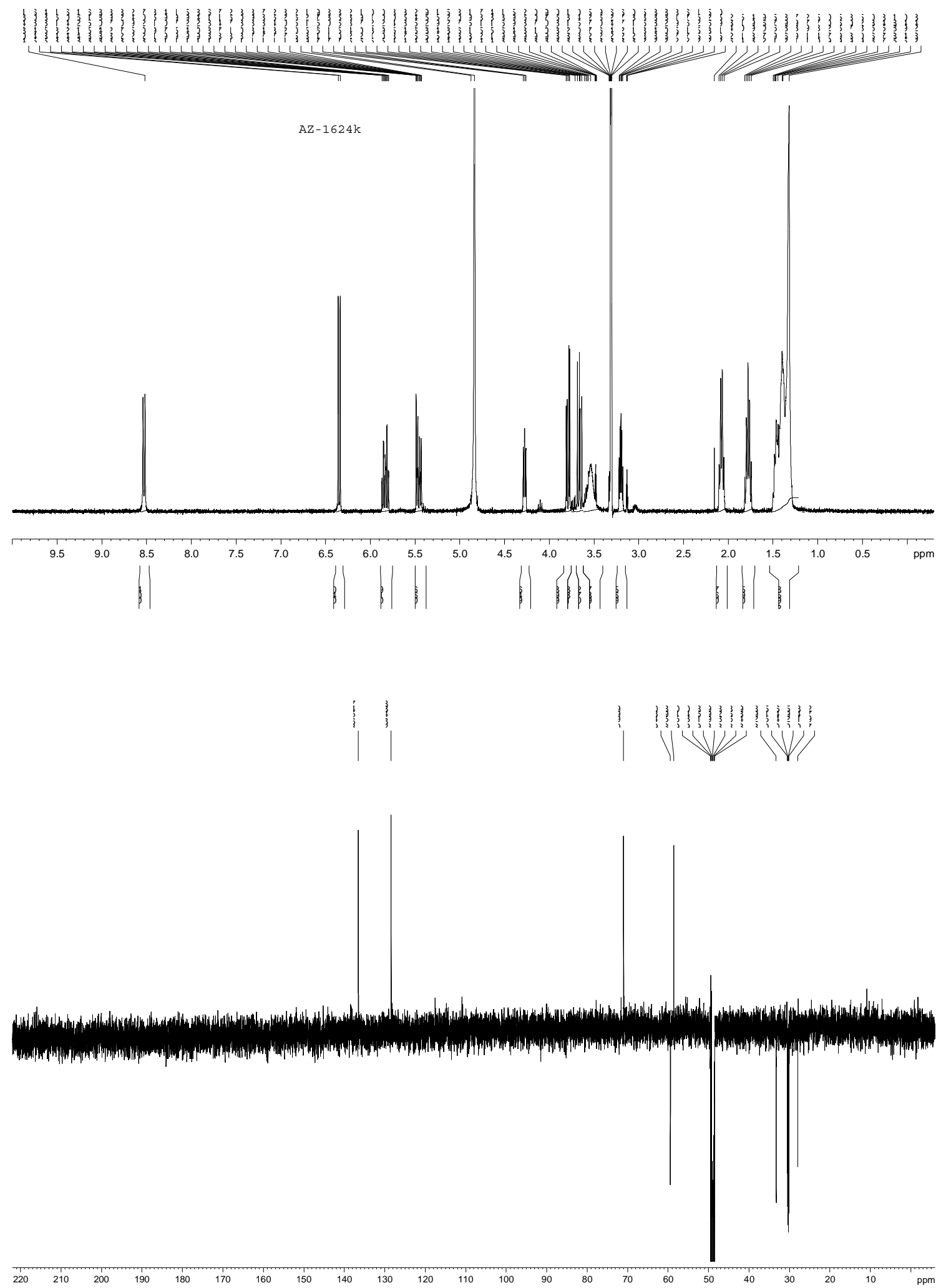
(E)-(2S,3R)-2-Amino-9-(4,4-Difluoro-5,7-dimethyl-4-bora-3a,4a-diaza-s-indacen-3)-non-4ene-1,3-diol $\mathbf{1 1 b}$
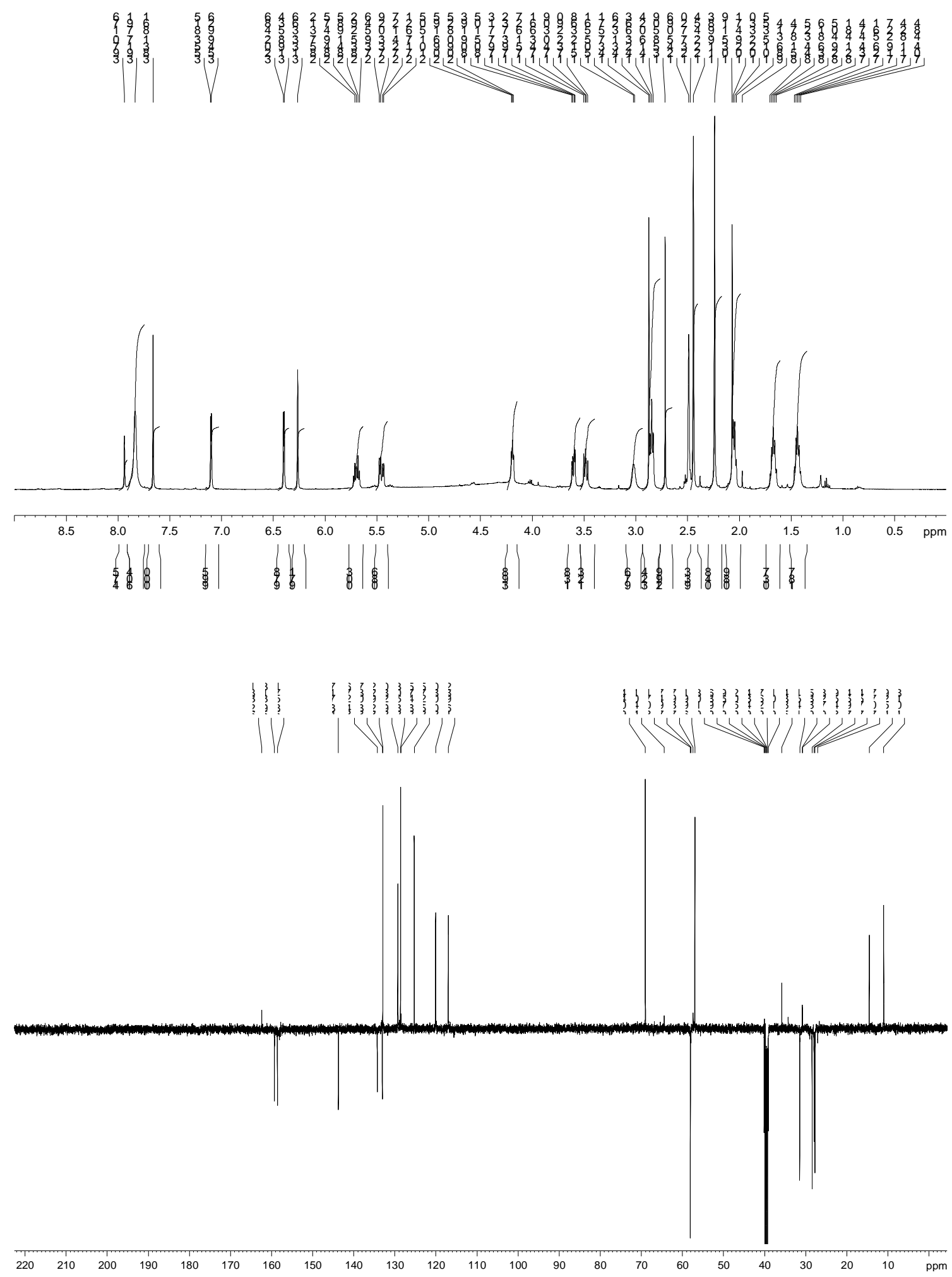
(E)-(2S,3R)-2-Amino-14-(4,4-difluoro-5,7-dimethyl-4-bora-3a,4a-diaza-s-indacen-3)-

tetradec-4-ene-1,3-diol 11c
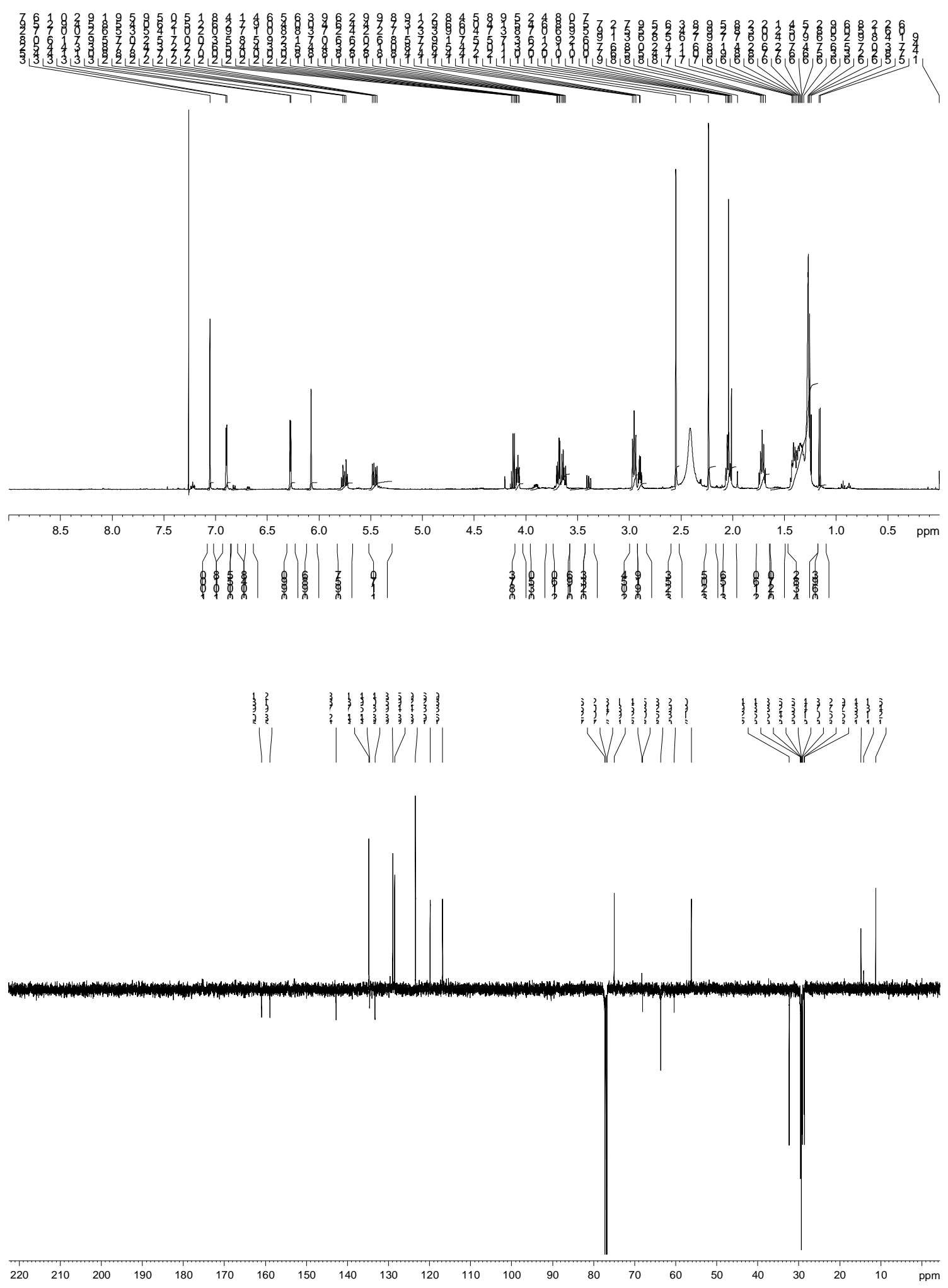
(E)-(2S,3R)-2-Amino-13-(4,4-Difluoro-1,3,5,7-tetramethyl-4-bora-3a,4a-diaza-s-indacene-

8)-tridec-4-ene-1,3-diol 11d

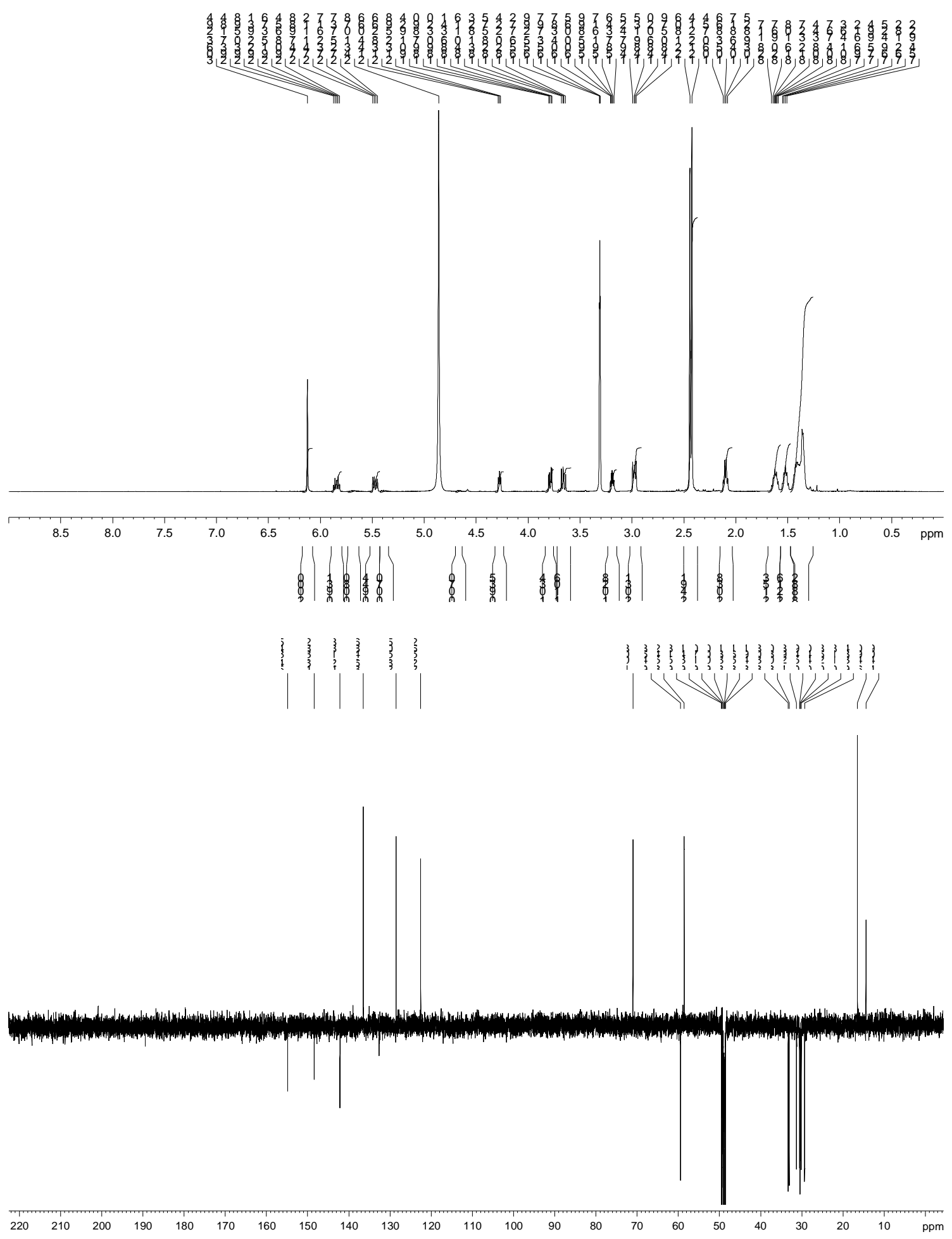




\section{Quantitative HPLC-analysis of compounds 11b-d}

Determination of fluorescence-purity was carried out on a Hewlett-Packard 1090M system using a Phenomenex Gemini C18 100*4 mm; $5 \mu \mathrm{m}$ column. Fluorophore was excited at $\lambda=$ $502 \mathrm{~nm}$ and emission was measured at $\lambda=515 \mathrm{~nm}$. Determination of UV-purity was carried out on a Agilent 1100 system using a XTerra RP18 4.6*50 mm, $3.5 \mu \mathrm{m}$ column. In both cases a gradient of acetonitrile in $10 \mathrm{mM}$ phosphate-buffer (adjusted to $\mathrm{pH}$ 5) starting from 5\% of acetonitrile to $95 \%$ within 8 min was used.

\section{HPLC spectra of compound $\mathbf{1 1 b}$}

Fluorescence detection, excitation wavelength $\lambda=502 \mathrm{~nm}$; purity $97.9 \%$ (according to integration)

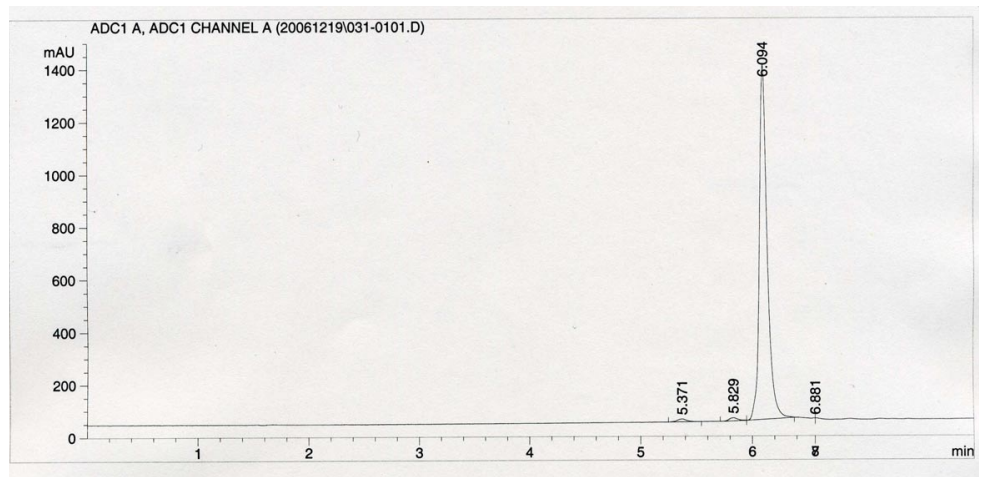


UV-detection, $\lambda=220 \mathrm{~nm}$, purity $98.2 \%$ (according to integration)

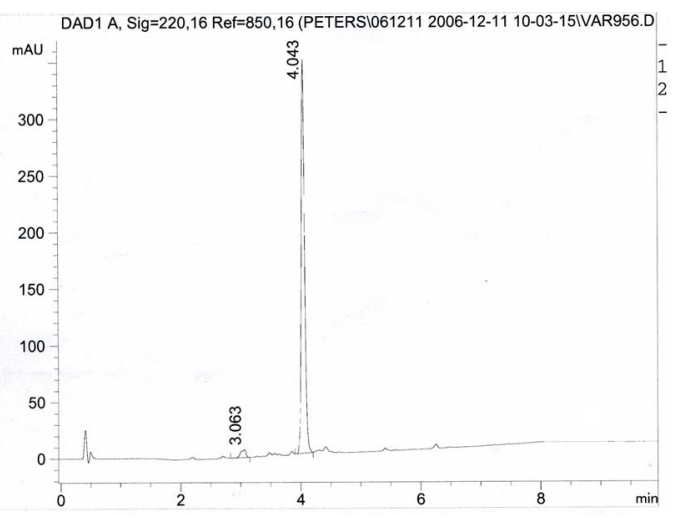

\section{HPLC spectra of compound 11c}

Fluorescence detection, excitation wavelength $\lambda=502 \mathrm{~nm}$; purity $97.9 \%$ (according to integration)

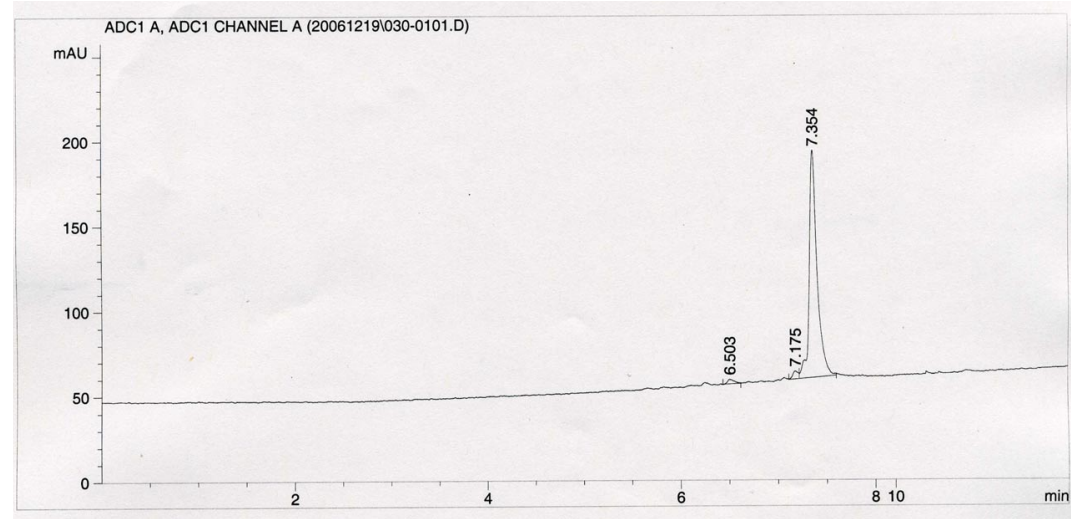

UV-detection, $\lambda=220 \mathrm{~nm}$, purity $98.2 \%$ (according to integration)

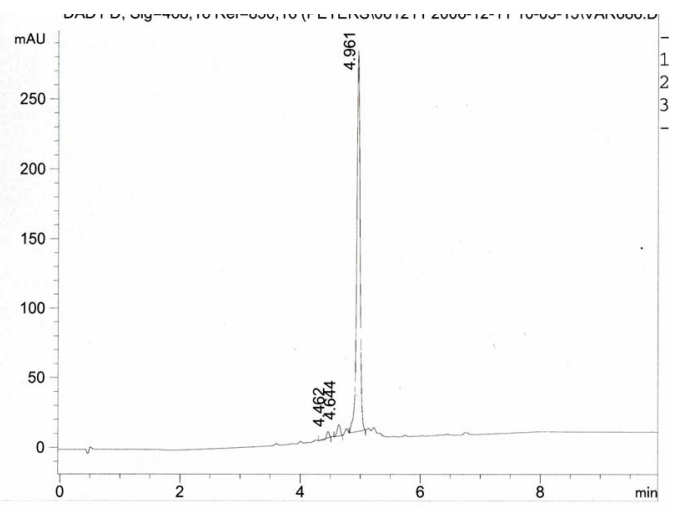




\section{HPLC spectra of compound 11d}

Fluorescence detection, excitation wavelength $\lambda=502 \mathrm{~nm}$; purity $96.8 \%$ (according to integration)

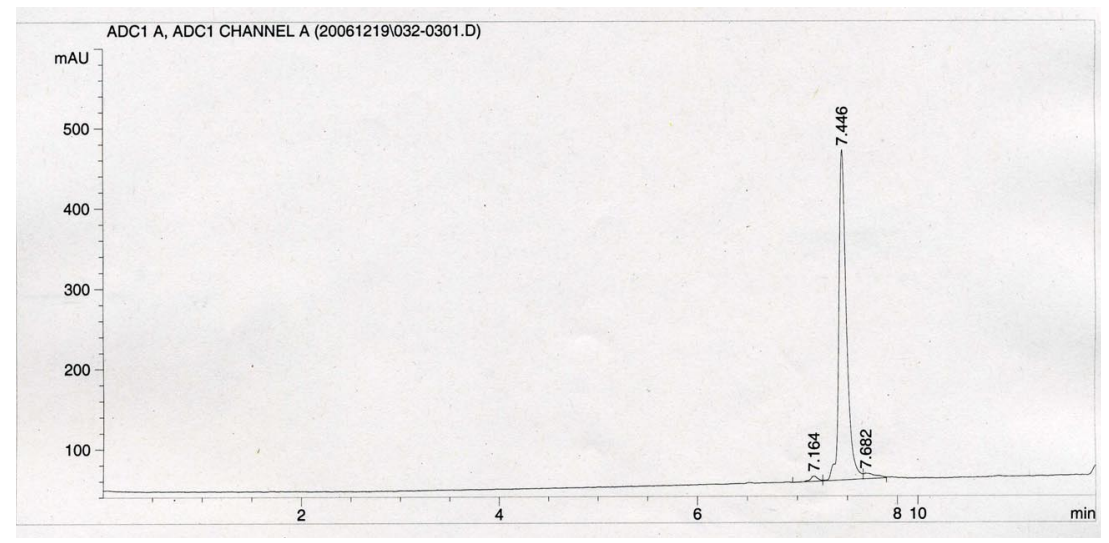

UV-detection, $\lambda=220 \mathrm{~nm}$, purity $97.1 \%$ (according to integration)

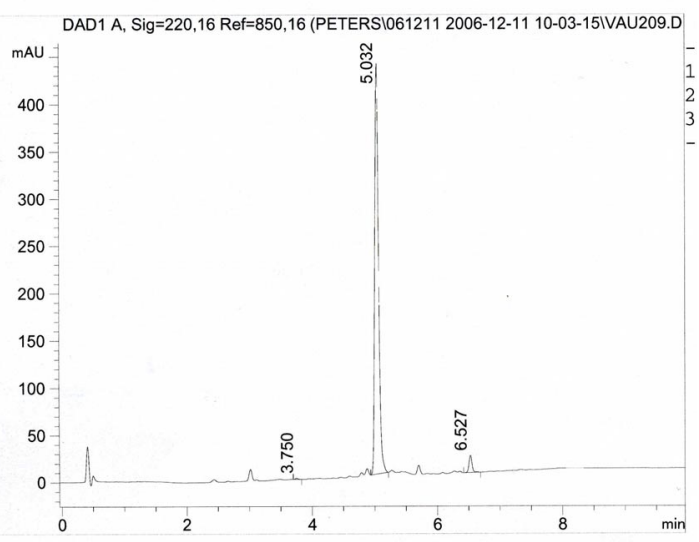

